\title{
Etudes expérimentales et numériques de la propagation des vagues à travers un réseau de cylindres verticaux en vue de la conception d'un système atténuateur de houle inspiré des racines de mangrove
}

\section{Michel BENOIT ${ }^{1}$, Alexis BEUDIN ${ }^{2}$, Julien DALLE ${ }^{3}$, Olivier KIMMOUN ${ }^{1}$, Fabien REMY ${ }^{1}$, Benoit WAELES ${ }^{2}$, Philippe BLANC ${ }^{4}$}

\section{Aix Marseille Univ, CNRS, Centrale Marseille, Irphé - UMR 7342, 49 rue Frédéric} Joliot-Curie, BP 146, 13384 Marseille Cedex 13, France.

michel.benoit@centrale-marseille.fr

2. BW-CGC, 253 Rue du Commandant Groix, 29200 Brest, France.

alexis.beudin@bw-cgc.fr

3. Seaboost, 889 Rue de la Vieille Poste, 34000 Montpellier, France.

julien.dalle@seaboost.fr

4. TOTAL, Avenue Larribau, 64018 Pau, France.

philippe.blanc@total.com

\section{Résumé :}

Le projet ROOT vise à concevoir un ouvrage innovant biomimétique de la géométrie des systèmes racinaires de palétuviers pour réhabiliter les forêts naturelles de mangrove côtières par réhabilitation préalable du contexte hydrosédimentaire. Dans le cadre du développement de cette solution, on schématise dans un premier temps ce système racinaire par un réseau de cylindres verticaux de section cylindrique. Des mesures en laboratoire (plateau mobile hexapode et canal à houle) et des simulations numériques ont été mises en œuvre pour étudier l'influence de diverses caractéristiques structurelles du système racinaire sur l'atténuation des vagues : diamètre des cylindres, tortuosité, rugosité, emprise de l'ouvrage, et porosité notamment. Les essais en hexapode ont permis d'évaluer des coefficients de traînée $C_{D}$ représentatifs des réseaux de cylindres, puis d'examiner l'influence de différents paramètres sur les valeurs de $\mathrm{C}_{\mathrm{D}}$. Ensuite, différentes valeurs de porosité, de diamètre des cylindres et de longueur du champ ont été testées en canal. En parallèle, trois modèles numériques de propagation de vagues (deux de type déterministes et un de type spectral) ont été mis en œuvre et comparés. Ils intègrent un terme dissipatif dépendant du réseau de cylindres, et déterminé à partir de la puissance des forces de traînée sur les cylindres. L'article présente les essais expérimentaux menés, et une vue d'ensemble des approches de modélisation testées, assortie d'une discussion sur l'analyse des processus physiques à l'œuvre, les résultats des différents modèles numériques testés et l'exposé de perspectives pour les étapes suivantes de développement du système ROOT. 


\section{Thème 4 - Ouvrages portuaires, offshore et de plaisance}

\section{Mots-clés :}

Atténuation des vagues, Mangrove, Génie côtier écologique, Modèle physique, Modèle numérique, Canal à houle, Réseau de cylindres, Restauration écologique.

\section{Introduction}

Le projet ROOT, développé par Seaboost, vise à concevoir un ouvrage innovant biomimétique de la géométrie des systèmes racinaires de palétuviers pour réhabiliter les forêts naturelles de mangrove côtières (COGNAT et al., 2020), et ce par réhabilitation d'un contexte hydrosédimentaire approprié. Dans le cadre du développement de cette solution, une étude hydrodynamique a été menée pour analyser les effets d'un réseau de cylindres verticaux, représentatif d'un système racinaire de mangrove, sur la propagation des vagues en eau peu profonde.

Cet article vise à présenter une vue d'ensemble des essais expérimentaux menés et des approches de modélisation testées, avec des comparaisons entre les mesures et les résultats de modèles, assortie d'une discussion sur l'analyse des processus à l'œuvre.

\section{Matériels et méthodes mis en œuvre}

\subsection{Tests en modèle physique}

Deux types de tests en modèle physique à échelle réduite ont été réalisés à Centrale Marseille/Irphé : d'abord sur plateau mobile hexapode, puis en canal à houle.

\subsubsection{Tests en hexapode}

$\mathrm{Au}$ cours des tests en hexapode, une cuve parallélépipédique contenant un réseau de cylindres verticaux est fixée sur le plateau de l'hexapode (figure 1). Cette cuve est soumise à des mouvements de translations de type sinusoïdal, en balayant un intervalle de fréquences d'excitation avec une faible amplitude de mouvement. L'élévation de surface libre est mesurée sur les parois intérieures de la cuve, ce qui permet de dégager une fonction de transfert entre le mouvement d'excitation et l'élévation de surface libre en fonction de la fréquence d'excitation. Un modèle mathématique potentiel linéarisé comportant un terme d'amortissant de type "traînée" (MOLIN \& REMY, 2016) est ensuite appliqué à ces mesures. Il permet d'estimer un coefficient de traînée $C_{D}$ caractéristique du réseau de cylindres (plus $C_{D}$ est grand, plus l'ouvrage dissipe l'énergie des vagues). Réalisés à une échelle $1 / 2.5$, ces essais ont permis d'explorer les effets sur le $\mathrm{C}_{\mathrm{D}}$ du diamètre $\mathrm{D}$ et de la densité des cylindres $\mathrm{N}$ (ou de la porosité du milieu définie par $\left.\tau=1-\mathrm{N} \pi \mathrm{D}^{2} / 4\right)$, de la tortuosité et de la rugosité racinaire. L'ensemble des configurations testées en hexapode est résumé dans le tableau 1. 


\section{XVIèmes Journées Nationales Génie Côtier - Génie Civil \\ Le Havre 2020}

\subsubsection{Tests en canal à houle}

Les tests en canal à houle à l'échelle 1/16 (figure 2) ont permis d'évaluer la performance d'atténuation de houle en fonction du diamètre racinaire $\mathrm{D}$, de la porosité de l'ouvrage $\tau$, $\mathrm{du}$ caractère homogène ou variable en espace de cette porosité, et de la largeur de l'ouvrage d. L'ensemble des configurations testées en canal est résumé dans le tableau 2. La configuration 1 est considérée comme la configuration "de référence".

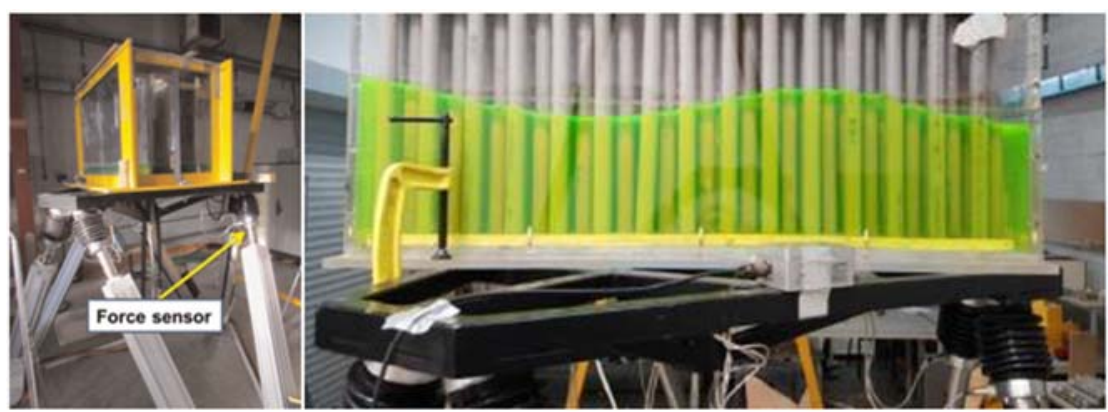

Figure 1. Vue générale de l'hexapode et exemple de test sur un réseau de cylindres réalisé à Centrale Marseille/Irphé.

Tableau 1. Synthèse des configurations testées en hexapode.

\begin{tabular}{|c|c|c|c|c|c|}
\cline { 3 - 5 } \multicolumn{1}{c|}{} & \multicolumn{5}{|c|}{ Propriétés de l'ouvrage } \\
\hline \multirow{3}{*}{ Config. } & $\begin{array}{c}\text { Porosité de } \\
\text { l'ouvrage } \tau[\%]\end{array}$ & $\begin{array}{c}\text { Diamètre des cylindres } \\
\text { [mm] en } \\
\text { hexapode }\end{array}$ & $\begin{array}{c}\text { D [mm] } \\
\text { au réel }\end{array}$ & $\begin{array}{c}\text { Tortuosité des } \\
\text { cylindres }\end{array}$ & $\begin{array}{c}\text { Rugosité des } \\
\text { cylindres }\end{array}$ \\
\cline { 3 - 5 } & 80 & 20 & 50 & rectilignes & lisses \\
\hline $80-32$ & $\mathbf{8 0}$ & $\mathbf{3 2}$ & $\mathbf{8 0}$ & rectilignes & lisses \\
\hline $90-50$ & 80 & 50 & 125 & rectilignes & lisses \\
\hline $80-63$ & 80 & 63 & 157.5 & rectilignes & lisses \\
\hline $70-32$ & 70 & 32 & 80 & rectilignes & lisses \\
\hline $90-32$ & 90 & 32 & 80 & rectilignes & lisses \\
\hline $80-32 R$ & 80 & 32 & 80 & rectilignes & rugueuses \\
\hline $80-32 T$ & 80 & 32 & 80 & tortueuses & lisses \\
\hline
\end{tabular}
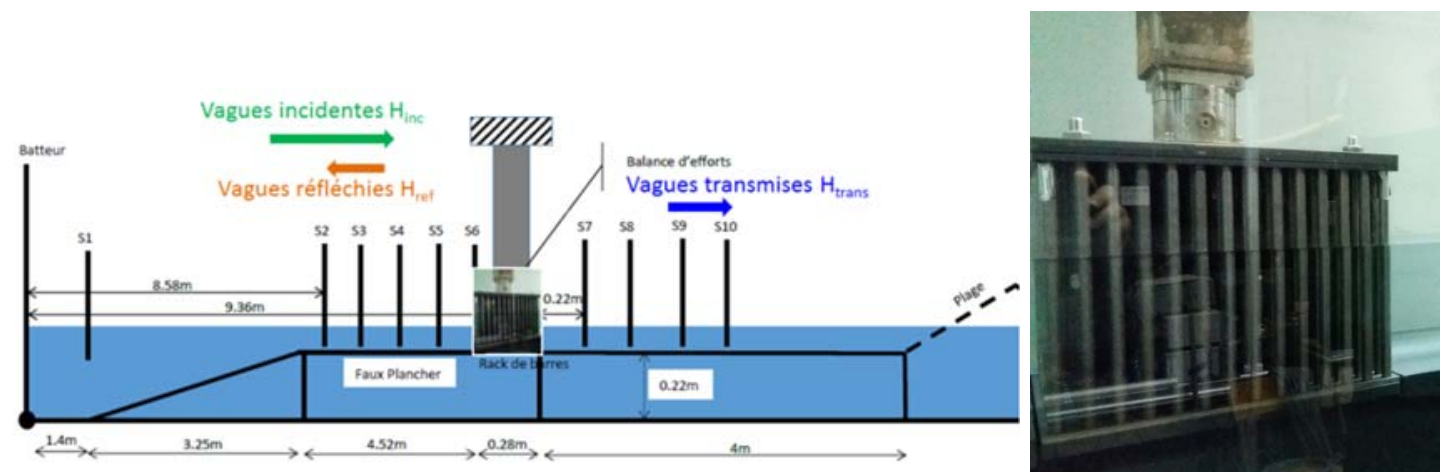

Figure 2. Gauche : schéma du canal à houle (pas à l'échelle) et positions des sondes de type résistive (S1 à S10). Droite : vue d'un module dans le canal à houle. 


\section{Thème 4 - Ouvrages portuaires, offshore et de plaisance}

\subsection{Modélisations numériques}

Les modélisations numériques ont permis d'étendre le champ expérimental à des configurations qui n'ont pas pu être testées sur modèle physique. Trois modèles ont été testés, reposant tous trois sur l'hypothèse que l'atténuation de l'énergie des vagues dans le réseau de cylindres est (uniquement ou principalement, suivant le modèle) due à la puissance dissipée par la force de traînée sur les cylindres.

Tableau 2. Synthèse des configurations testées en canal à houle.

\begin{tabular}{|c|c|c|c|c|c|c|}
\hline & \multicolumn{3}{|c|}{ Module 1 } & \multicolumn{3}{c|}{ Module 2 } \\
\hline Config. & $\begin{array}{c}\text { Porosité } \tau \\
{[\%]}\end{array}$ & $\begin{array}{c}\text { Diamètre des cylindres } \\
\text { [cm] en } \\
\text { canal }\end{array}$ & $\begin{array}{c}\text { D [cm] au } \\
\text { réel }\end{array}$ & $\begin{array}{c}\text { Porosité } \tau \\
{[\%]}\end{array}$ & $\begin{array}{c}\text { Diamètre des cylindres } \\
{[\mathrm{cm}] \text { en }} \\
\text { canal }\end{array}$ & $\begin{array}{c}\text { D [cm] au } \\
\text { réel }\end{array}$ \\
\hline 1 & & & & 80 & 0.5 & 8 \\
\hline 2 & & & & 80 & 1.0 & 16 \\
\hline 3 & & & & 90 & 0.5 & 8 \\
\hline 4 & & & & 70 & 0.5 & 8 \\
\hline 5 & 90 & 0.5 & 8 & 70 & 0.5 & 8 \\
\hline 6 & 80 & 0.5 & 8 & 80 & 0.5 & 8 \\
\hline
\end{tabular}

\subsubsection{Formulation du paramètre $C_{D}$}

DALRYMPLE et al. (1984) ont établi une expression du taux de dissipation de l'énergie des vagues (supposées monochromatiques et progressives) due aux effets de traînée pour un ensemble de cylindres verticaux. Cette modélisation se base principalement sur la spécification d'un coefficient de traînée $C_{D}$. Ce coefficient est dépendant de la nature de l'ouvrage, mais aussi des caractéristiques des vagues. Différentes formulations ont été proposées dans la littérature pour estimer ce $\mathrm{C}_{\mathrm{D}}$ en fonction de nombres adimensionnels de type Reynolds ou Keulegan-Carpenter (e.g., HU et al., 2014 ; OZEREN et al., 2014 ; LIU et al., 2015 ; LUHAR \& NEPF, 2016 ; CHEN et al., 2018). Certaines d'entre elles ont été appliquées dans la présente étude pour évaluer la sensibilité des résultats à l'expression du $\mathrm{C}_{\mathrm{D}}$.

\subsubsection{Modèles numériques mis en œuvre}

Trois modèles numériques de propagation de vagues ont été utilisés :

a) PREMISE : modèle déterministe (à résolution de phase) développé à Centrale Marseille/Irphé, basé sur l'équation de Berkhoff munie d'un terme dissipatif dérivé de la formulation de DALRYMPLE et al. (1984). Dans sa version actuelle, ce modèle ne traite que des vagues monochromatiques en une dimension d'espace horizontale. Ce modèle inclut les effets de réflexion-diffraction, et offre l'avantage de pouvoir réaliser aisément de nombreux tests de sensibilité à différents paramètres. 


\section{XVI'mes Journées Nationales Génie Côtier - Génie Civil \\ Le Havre 2020}

b) SWAN : modèle spectral (à phases moyennées) développé à TU Delft (NL) intégrant un terme de dissipation énergétique par macro-rugosité (e.g., tiges de végétation) dont la formulation (SUZUKI et al., 2011) découle du modèle proposé par DALRYMPLE et al. (1984). Prenant en compte le caractère irrégulier et directionnel des vagues, il est indiqué pour des applications à grande échelle, mais ne prend pas en compte les effets de réflexion et diffraction.

c) SWASH : modèle déterministe, également développé à TU Delft (NL), basé sur les équations en eau peu profonde avec un terme de pression non-hydrostatique permettant de résoudre la structure verticale de l'écoulement. En plus d'un terme de dissipation par la force de traînée (comme pour les deux modèles précédents), le module végétation de SWASH (SUZUKI et al., 2019) intègre l'influence de la force d'inertie (cf. MORISON et al., 1950) et l'effet de la porosité (en résolvant la vitesse d'écoulement entre les pieux). Le modèle est ici utilisé avec deux couches verticales.

\subsection{Méthode d'analyse des résultats}

L'analyse des tests des modèles et les comparaisons aux essais en canal à vagues s'appuient sur les valeurs de 3 coefficients : $\mathrm{C}_{\text {trans }}=\mathrm{H}_{\text {trans }} / \mathrm{H}_{\text {inc }}$ qui caractérise la transmission des vagues à travers l'ouvrage, $\mathrm{C}_{\text {ref }}=\mathrm{H}_{\text {ref }} / \mathrm{H}_{\text {inc }}$ qui caractérise la réflexion des vagues sur l'ouvrage et $\mathrm{C}_{\mathrm{dis}}=\left(1-\mathrm{C}_{\text {trans }}{ }^{2}-\mathrm{C}_{\mathrm{ref}}{ }^{2}\right)^{1 / 2}$ qui caractérise la dissipation. En terme énergétique, on a la relation suivante sur ces coefficients élevés au carré :

$\mathrm{C}_{\text {trans }}{ }^{2}+\mathrm{Cref}^{2}+\mathrm{C}_{\text {dis }}{ }^{2}=1$

Les modèles sont notamment utilisés pour examiner l'impact de la variation d'un paramètre sur la performance de l'ouvrage dans divers contextes.

\section{Résultats}

\subsection{Valeurs du coefficient de traînée $C_{D}$ à partir des tests en hexapode}

Les valeurs de $C_{D}$ obtenues à l'issue des tests en hexapode sont synthétisées dans le tableau 3. On peut retenir que :

a) à porosité $\tau$ et hauteur d'eau $h$ fixées, $C_{D}$ augmente quand le diamètre racinaire $D$ diminue (i.e. lorsque le nombre de cylindres par unité de surface augmente).

b) à diamètre $\mathrm{D}$ et hauteur d'eau $\mathrm{h}$ fixés, $\mathrm{C}_{\mathrm{D}}$ augmente quand la porosité $\tau$ diminue.

c) à porosité $\tau$ et diamètre $\mathrm{D}$ fixés, $\mathrm{C}_{\mathrm{D}}$ augmente quand la hauteur d'eau $\mathrm{h}$ diminue.

d) $C_{D}$ augmente significativement si la rugosité des cylindres est augmentée. C'est la caractéristique qui a l'effet le plus marqué sur la valeur de $C_{D}$, avec des valeurs de $C_{D}$ supérieures à 3 (par rapport à des valeurs autour de 2 pour des cylindres lisses).

e) CD semble augmenter avec la tortuosité, mais il n'est pas évident de comparer la valeur obtenue aux autres cas, car la porosité du milieu variait également sur ces essais. Par ailleurs, seulement deux cas exploratoires ont été testés durant cette phase. 
Thème 4 - Ouvrages portuaires, offshore et de plaisance

Tableau 3. Valeurs du coefficient $C_{D}$ obtenues à l'issue des tests en hexapode.

\begin{tabular}{|c|c|c|c|}
\hline & \multicolumn{3}{|c|}{ Hauteur d'eau } \\
\hline Config. & $h=120 \mathrm{~mm}$ & $h=235 \mathrm{~mm}$ & $h=370 \mathrm{~mm}$ \\
\hline $80-20$ & & 2.5 & 1.5 \\
\hline $80-32$ & 2.5 & 1.8 & 1.2 \\
\hline $80-50$ & 2.0 & 1.3 & 1.8 \\
\hline $80-63$ & & 2.5 (non-réaliste) & 1.5 \\
\hline $70-32$ & 2.0 & 2.0 & 3.5 \\
\hline $90-32$ & 2.5 & 1.5 & 1.5 (non-réaliste) \\
\hline $80-32 R$ & & 3.2 & 2.8 \\
\hline $80-32 T$ & & & \\
\hline
\end{tabular}

\subsection{Performance des différentes configurations testées en canal et effets d'échelle}

Les résultats des tests en canal, exprimés via les valeurs des coefficients $\mathrm{C}_{\text {trans }^{2}}{ }^{2} \mathrm{C}_{\text {ref }}{ }^{2}$, et $\mathrm{C}_{\mathrm{dis}}{ }^{2}$, sont résumés sur la figure 3 . Deux constats principaux en ressortent :

a) Les configurations testées permettent d'atteindre des taux de dissipation énergétique de 45 à $70 \%$, avec des réflexions variant de 3 à $20 \%$ en moyenne.

b) Les effets d'échelle sont plus importants qu'escomptés, et on obtient sur les hauteurs de vague les plus faibles des résultats difficiles à extrapoler en situation réelle (écarts importants entre les valeurs de $\mathrm{C}_{\mathrm{D}}$ issues de formules de la littérature et ceux obtenus sur une partie des tests).

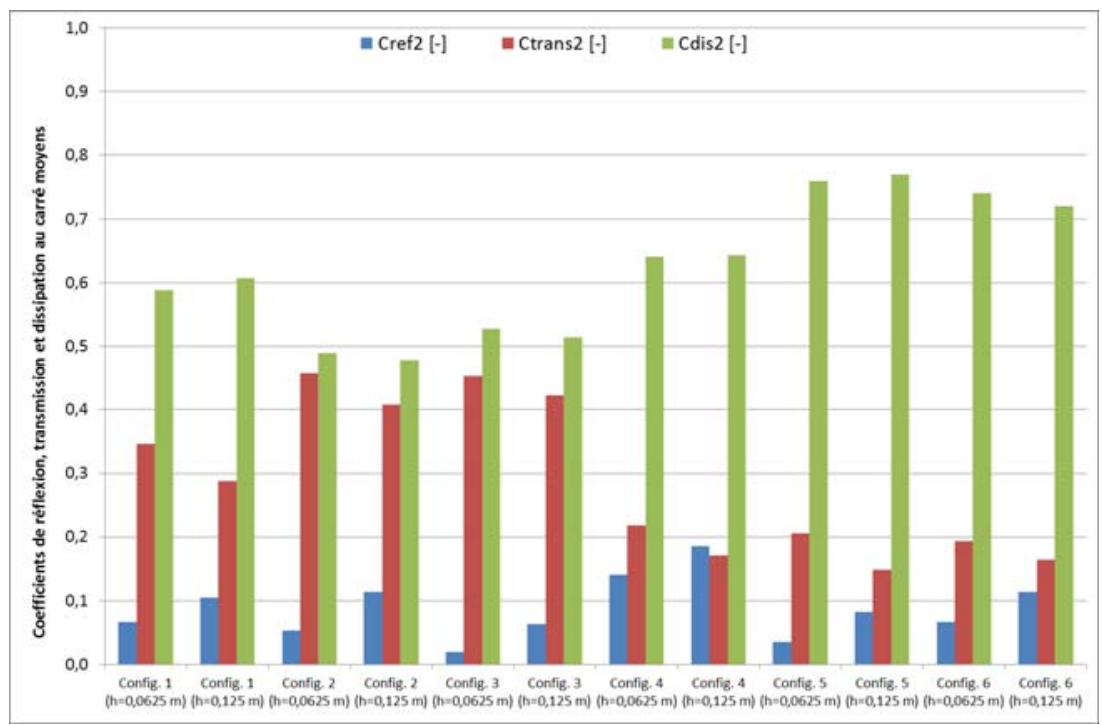

Figure 3. Valeurs des carrés des coefficients de réflexion, transmission et dissipation pour chaque configuration et les deux hauteurs d'eau testées en canal à houle.

\subsection{Apports des modélisations numériques}

Les tests en modèles numériques ont permis d'explorer des configurations complémentaires à celles testées en canal à houle et de répondre à des questions qui n'ont 


\section{XVI'̀mes Journées Nationales Génie Côtier - Génie Civil \\ Le Havre 2020}

pas pu être abordées sur ces mêmes tests. On présente ci-après des résultats de tests de sensibilité réalisés avec PREMISE (en vagues régulières). Sauf indication contraire, ces tests sont réalisés à partir d'une configuration de base suivante : hauteur d'eau $h=1 \mathrm{~m}$, hauteur de vague incidente $H=0.25 \mathrm{~m}$, période des vagues $\mathrm{T}=7 \mathrm{~s}$, diamètre des pieux $\mathrm{D}=8$ $\mathrm{cm}$, hauteur des pieux $\mathrm{s}=\mathrm{h}=1 \mathrm{~m}$ (cylindres émergents), porosité du réseau $\tau=80 \%$ (soit une densité de pieux de $\mathrm{N}=40$ pieux par $\mathrm{m}^{2}$ ), longueur du réseau $\mathrm{d}=5 \mathrm{~m}$, à partir de $\mathrm{x}=0$, sur un domaine de calcul allant de de $\mathrm{x}=-30 \mathrm{~m}$ à $+30 \mathrm{~m}$.

\subsubsection{Influence de la porosité en deçà des porosités testées en canal}

On observe une évolution non-linéaire de la performance d'atténuation des vagues par l'ouvrage avec la porosité $\tau$. En passant d'une porosité de $90 \%$ à $70 \%$, la hauteur des vagues derrière l'ouvrage passe de $72 \%$ à $51 \%$ (figure 4 ). Si on diminue encore la porosité à $50 \%$, on atteint une hauteur des vagues derrière l'ouvrage de $40 \%$ de la hauteur des vagues incidentes. Cela signifie qu'on a un écart de performance 3 fois plus élevé entre $90 \%$ et $70 \%$ de porosité qu'entre $70 \%$ et $50 \%$ de porosité.

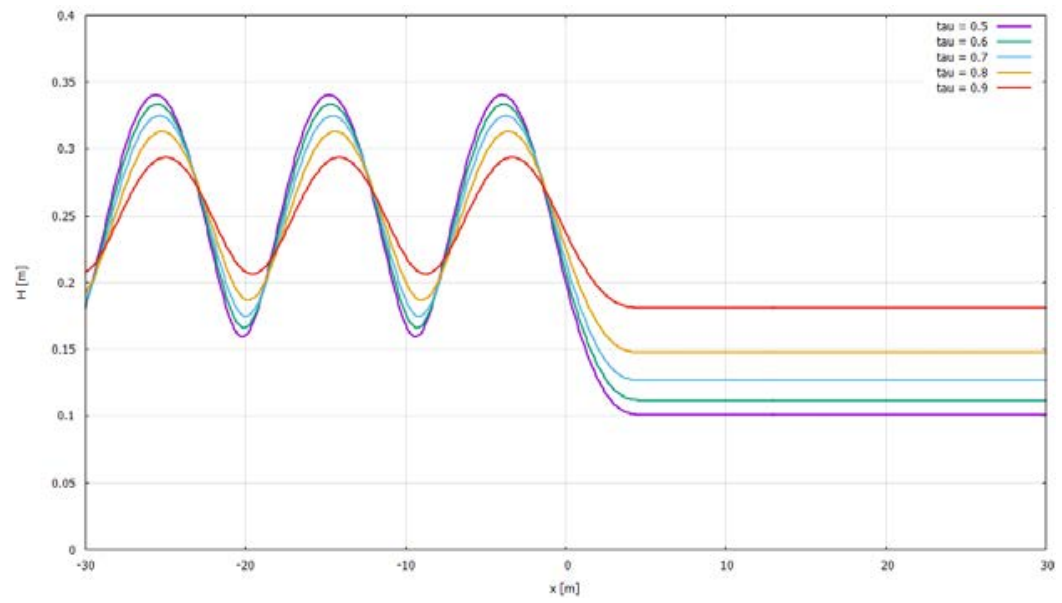

Figure 4. Profils spatiaux de hauteur des vagues calculés avec PREMISE pour différentes porosités par rapport à la configuration de base (voir texte) (NB : le réseau de cylindres occupe l'intervalle en $x$ de 0 à $5 \mathrm{~m}$ ).

\subsubsection{Effet d'une largeur d'ouvrage significativement supérieure}

En élargissant l'ouvrage par rapport aux valeurs testées en canal, c'est-à-dire en augmentant la distance que doivent parcourir les vagues dans l'ouvrage, on s'aperçoit que l'on atteint assez rapidement une tendance asymptotique (figure 5). Globalement, on atténue environ $40 \%$ de la hauteur des vagues sur les 5 premiers mètres (porosité $80 \%$ diamètre racinaire $8 \mathrm{~cm}$ ), et $60 \%$ sur les 10 premiers mètres. Doubler la largeur de l'ouvrage de $10 \mathrm{~m}$ à $20 \mathrm{~m}$ permet de passer de $60 \%$ d'atténuation de la hauteur des vagues à $72 \%$. On dissipe avec un ouvrage de $20 \mathrm{~m}$ de large $94 \%$ de l'énergie des vagues incidents, contre $87 \%$ avec un ouvrage de $10 \mathrm{~m}$ de large. 


\section{Thème 4 - Ouvrages portuaires, offshore et de plaisance}

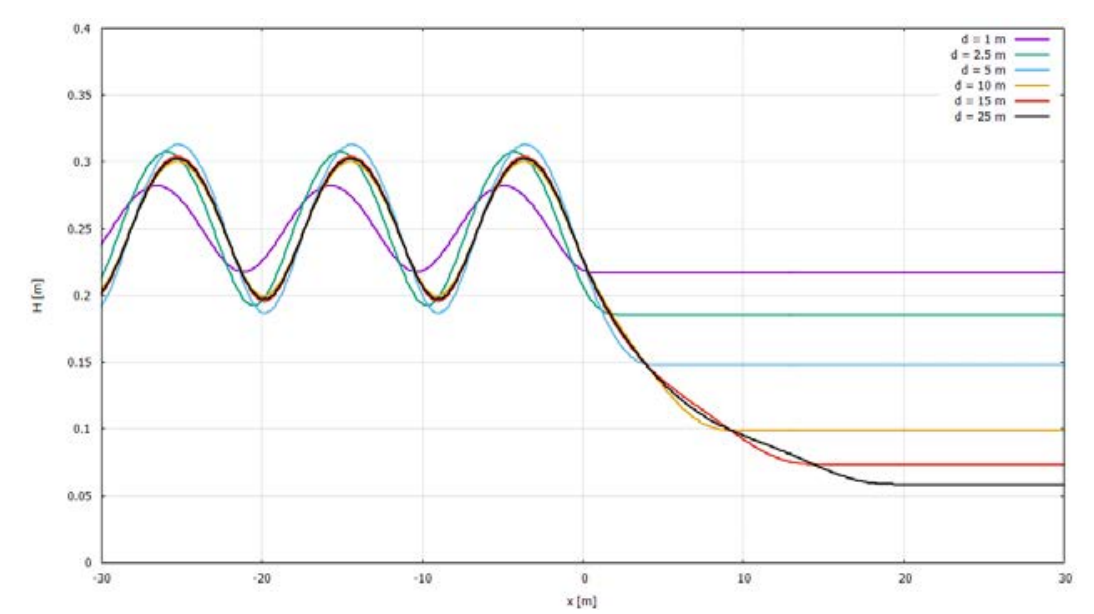

Figure 5. Profils spatiaux de hauteur des vagues calculés avec PREMISE pour différentes largeurs d'ouvrage d par rapport à la configuration de base (voir texte)

(NB : le réseau de cylindres débute dans tous les cas à l'abscisse $x=0$ ).

\subsubsection{Influence de la hauteur des racines dans la colonne d'eau}

La hauteur des racines, notée s, a un effet majeur sur la performance d'atténuation de l'ouvrage (figure 6). La performance décroît de manière significative lorsque les cylindres n'occupent plus l'ensemble de la colonne d'eau. Ainsi, on dissiperait $72 \%$ de l'énergie de la houle avec un ouvrage émergeant $(\mathrm{s}=\mathrm{h}=1 \mathrm{~m})$ contre seulement $32 \%$ avec un ouvrage occupant $50 \%$ de la colonne d'eau ( $(\mathrm{s}=0.5 \mathrm{~m}$ ).

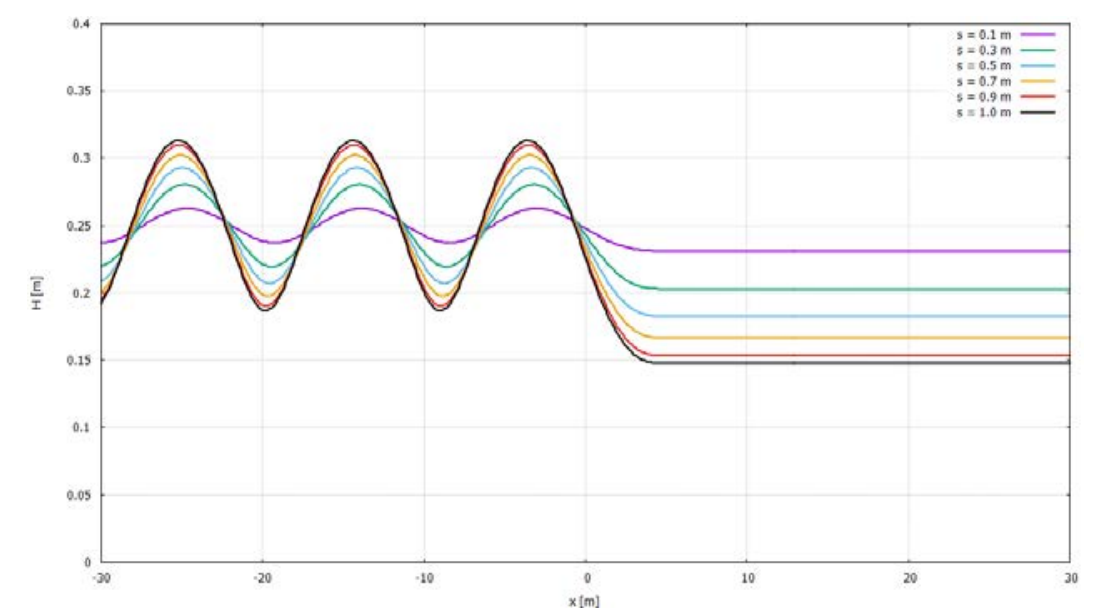

Figure 6. Profils spatiaux de hauteur des vagues calculés avec PREMISE pour différentes hauteurs de cylindres s par rapport à la configuration de base (voir texte) (NB : le réseau de cylindres occupe l'intervalle en $x$ de 0 à $5 \mathrm{~m}$ ). 


\section{XVI èmes Journées Nationales Génie Côtier - Génie Civil \\ Le Havre 2020}

\subsubsection{Efficacité de l'ouvrage pour des hauteurs de vagues supérieures}

La hauteur des vagues a été variée de 5 à $50 \mathrm{~cm}$ (toujours avec $\mathrm{h}=1 \mathrm{~m}$ ). On observe une influence toujours présente de la structure sur des vagues incidentes deux fois plus hautes que celles testées en canal, avec des taux d'atténuation des vagues pouvant atteindre $70 \%$ pour des vagues de $0.5 \mathrm{~m}$ de haut (figure 7). Il est possible qu'une valeur limite soit atteinte au-delà de laquelle la performance de la structure décroît significativement, mais nous ne l'avons pas atteinte dans le cadre de ces simulations.

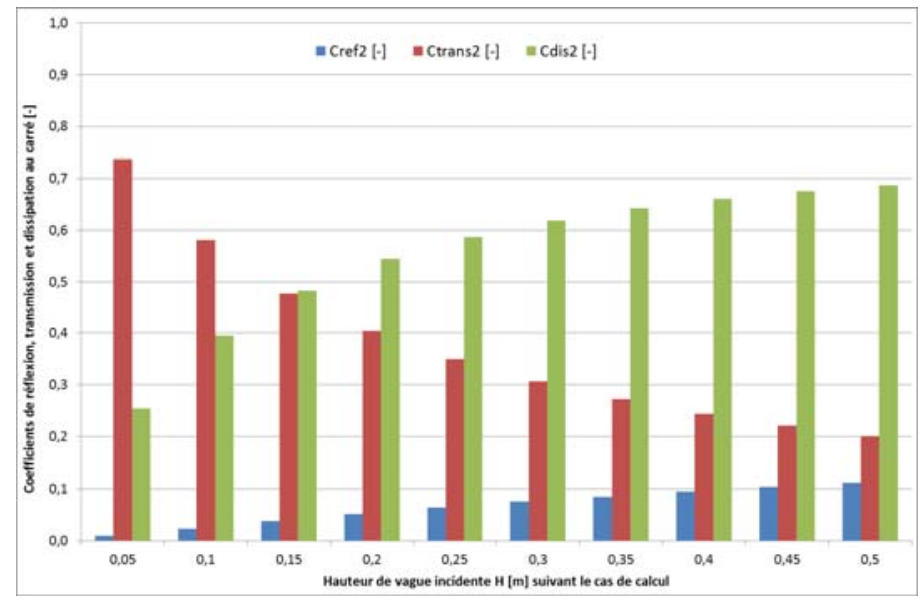

Figure 7. Performance d'atténuation de la houle de l'ouvrage en fonction de la hauteur de la houle incidente calculée avec PREMISE par rapport à la configuration de base.

\subsubsection{Sensibilité des résultats à la formulation $d u C_{D}$}

Différentes formules et valeurs issues de la littérature ont été appliquées pour évaluer dans quelle mesure la variation du $C_{D}$ impacte les résultats des tests (figure 8). L'énergie dissipée varie de $45 \%$ à $65 \%$ environ selon que le $\mathrm{C}_{\mathrm{D}}$ est de l'ordre de 1 ou de 3 . Il en est de même pour l'énergie transmise qui varie de $55 \%$ à $25 \%$ si le $C_{D}$ varie dans le même sens (de 1 à 3 ). Ces tests confirment que ce paramètre a donc une influence forte sur les résultats. Toutes les variations se font logiquement dans le même sens (cf. conservation de l'énergie). Cela signifie que la modalité de détermination du $C_{D}$ peut amener à revoir les résultats bruts des modèles, mais pas la comparaison de deux configurations l'une par rapport à l'autre, si elles sont testées avec le même $\mathrm{C}_{\mathrm{D}}$. Les constats restent donc valides, mais les écarts entre deux configurations pourraient être amoindris ou augmentés selon le mode de détermination du $\mathrm{C}_{\mathrm{D}}$.

Par ailleurs, il est intéressant de noter que, dans la configuration de pieux émergents, les deux modèles SWAN et SWASH fournissent des valeurs proches du coefficient de dissipation. La dissipation due à la force de traînée est donc le processus dominant. 


\section{Thème 4 - Ouvrages portuaires, offshore et de plaisance}

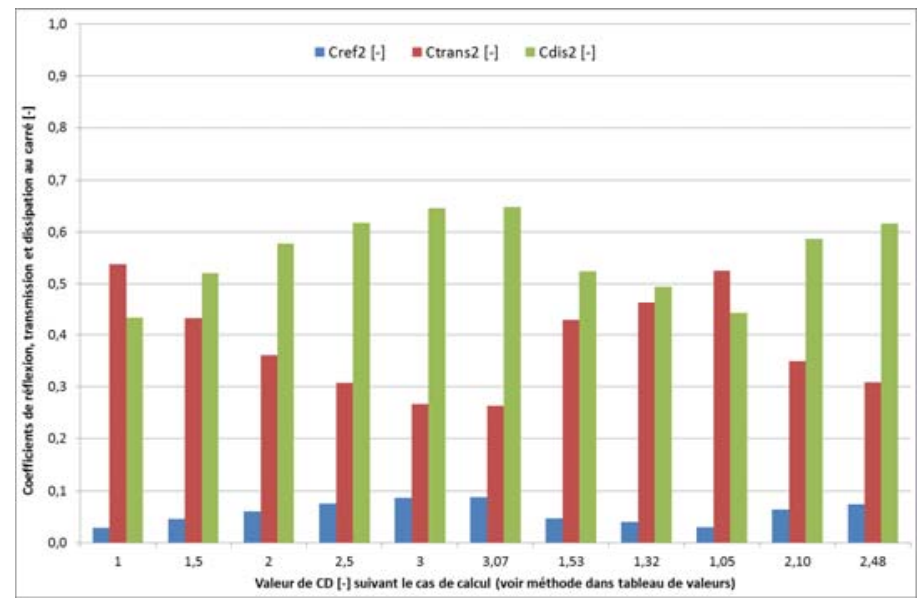

Figure 8. Influence de la valeur du $C_{D}$ (6 $C_{D}$ fixés, $6 C_{D}$ calculés) sur la performance d'atténuation de houle calculée avec PREMISE par rapport à la configuration de base.

\section{Conclusions et perspectives}

Les simulations réalisées avec les modèles numériques avaient pour objectif d'amener un premier éclairage sur l'intérêt de certains choix techniques comme la diminution de la porosité ou l'élargissement significatif de l'ouvrage dans la perspective d'un projet futur. Etant données les tendances homogènes dégagées par ces analyses et les écarts générés par les variations de $C_{D}$, le mode de détermination de ce coefficient ne devrait pas remettre en question les observations fondamentales. Par exemple, sur l'élargissement de l'ouvrage, on atténue dans tous les cas l'essentiel de l'énergie des vagues incidentes dans les 5 premiers mètres, que le $C_{D}$ soit de 1 ( $\sim 40 \%$ de l'énergie dissipée sur $\left.10 \mathrm{~m}\right)$ ou de 3 ( $\sim 60 \%$ de l'énergie dissipée sur $10 \mathrm{~m}$ ). Ainsi, dans la perspective du développement d'une solution atténuative de houle, on privilégiera dans un premier temps des configurations présentant une porosité dans la gamme de 70 à $90 \%$ et une largeur d'ouvrage d'une dizaine de mètres maximum.

La définition d'une méthodologie d'estimation d'un $\mathrm{C}_{\mathrm{D}}$ plus adapté aux applications cibles et calibré en situation réelle, si possible, est cependant une question qu'il convient de continuer à creuser, dans le cadre de la définition d'un modèle de simulation du déploiement de la solution ROOT sur un site précis.

Pour ce qui est des outils numériques, le code SWAN paraît une bonne option pour des simulations sur sites réels avec des domaines de plusieurs kilomètres de côté. Cependant, on rappelle que ce code ne permet pas de modéliser la réflexion ni la diffraction par le réseau de pieux. Au cas où ces processus se révèleraient importants (pour étudier les interactions de patchs de cylindres entre eux par exemple), le choix d'un modèle à résolution de phases (du type du modèle déterministe PREMISE ou de SWASH, par exemple) doit être privilégié.

Ainsi, dans la perspective de projets de restauration de zones de mangroves, et plus largement d'atténuation des phénomènes érosifs littoraux, ces résultats permettent 


\section{XVI èmes Journées Nationales Génie Côtier - Génie Civil \\ Le Havre 2020}

d'étayer, sous conditions, l'intérêt d'ouvrages perméables, tortueux, bio-inspirés et fondés sur la nature. Outre les performances que l'on met ici en évidence, outre l'intérêt de ces structures au regard des environnements sédimentaires et écosystèmes côtiers associés, les travaux à venir devraient permettre de modéliser les effets de tels ouvrages dans des contextes précis, et de dégager des pistes d'optimisation techniques et économiques applicables aux démarches d'adaptation au changement climatique.

\section{Références bibliographiques}

CHEN H., NI Y., LI Y., LIU F., OU S., SU M., PENG Y., HU Z., UIJTTEWAAL W., SUZUKI T. (2018). Deriving vegetation drag coefficients in combined wave-current flows by calibration and direct measurement methods. Advances in Water Resources, Vol. 122, pp 217-227. https://doi.org/10.1016/j.advwatres.2018.10.008

COGNAT M., DALlE J., GAVOILlE A., BEUDIN A., WAELES B., BENOIT M., BLANC P. (2020). Restauration de mangroves : développement d'une solution innovante et basée sur la nature (projet ROOT.), XVIème Journées Nationales Génie Côtier - Génie Civil, 8-10 décembre 2020, Le Havre, 10 p.

DALRYMPLE R.A., KIRBY J.T., HWANG P.A. (1984). Wave diffraction due to areas of energy dissipation. Journal of Waterway, Port, Coastal and Ocean, Vol. 110(1), pp 6779. https://doi.org/10.1061/(ASCE)0733-950X(1984)110:1(67)

HU Z., SUZUKI T., ZITMAN T., UITTEWAAL W., STIVE M. (2014). Laboratory study on wave dissipation by vegetation in combined current-wave flow. Coastal Engineering, Vol. 88, pp 131-142. https://doi.org/10.1016/j.coastaleng.2014.02.009

LIU P.L.-F., CHANG C.-W., MEI C.C., LOMONACO P., MARTIN F.L., MAZA M. (2015). Periodic water waves through an aquatic forest, Coastal Engineering, Vol. 96, pp 100-117. https://doi.org/10.1016/j.coastaleng.2014.11.002

LUHAR M., NEPF H. (2016). Wave-induced dynamics of flexible blades. Journal of Fluids and Structures, Vol. 61, pp 20-41. https://doi.org/10.1016/j.jfluidstructs.2015.11.007

MOLIN B., REMY F. (2016). Etude théorique et expérimentale du ballottement dans une cuve rectangulaire remplie de cylindres verticaux. $15^{\mathrm{èmes}}$ Journées de l'Hydrodynamique, Brest, $13 \mathrm{p}$.

MORISON J.R., O'BRIEN M.P., JOHNSON J.W., SCHAAF S.A. (1950). The force exerted by surface waves on piles. Petroleum Transactions. 189, pp 149-157.

SUZUKI T., ZIJLEMA M., BURGER B., MEIJER M., NARAYAN S. (2011). Wave dissipation by vegetation with layer schematization in SWAN. Coastal Engineering, Vol. 59, pp 64-71. https://doi.org/10.1016/j.coastaleng.2011.07.006

SUZUKI T., HU Z., KUMADA K., PHAN L.K., ZIJLEMA M. (2019). Non-hydrostatic modeling of drag, inertia and porous effects in wave propagation over dense vegetation fields. Coastal Engineering, Vol. 149, pp 49-64. https://doi.org/10.1016/j.coastaleng.2019.03.011 
Thème 4 - Ouvrages portuaires, offshore et de plaisance 